\title{
THERMAL DAMPING IN CAVITATING NOZZLE FLOWS
}

\author{
Can F. Delale \\ Department of Aeronautics and Astronautics, Istanbul Technical University, 80626 Maslak, Istanbul \\ and TÜBİTAK Feza Gürsey Institute, P.O. Box 6, 81220 Çengelköy, Istanbul, Turkey.
}

\begin{abstract}
Recent investigations of bubbly cavitating nozzle flows by Wang \& Brennen (1998) and by Delale et al. (2001), where various damping effects are lumped together in an adhoc manner, have shown flow instabilities that lead to flashing flow solutions. Here, we investigate the stabilizing effect of thermal damping on these instabilities. For this reason we consider the energy equation within the bubble, assumed to be composed of vapor and gas, in the uniform pressure approximation (similar to that given by Nigmatulin et al., 1981 and by Prosperetti, 1991). The partial vapor pressure is fixed by the vapor saturation pressure corresponding to the interface temperature, which is evaluated by the Plesset-Zwick ${ }^{5}$ formula assuming the thin boundary layer approximation within the liquid. Consequently, the partial gas pressure is evaluated by its relation to the heat flux through the interface in the uniform pressure approximation. The model is then coupled to the steady-state cavitating nozzle flow equations employed by Wang \& Brennen and by Delale et al., replacing the previously assumed polytropic law for the partial gas pressure. The instabilities arising from the use of the polytropic law for the gas pressure in steady cavitating nozzle flows are seen to be stabilized by thermal damping with or without the occurrence of bubbly shock waves.
\end{abstract}

\section{Introduction}

It is well-known that the pressure within the bubble affects bubble dynamics significantly. Thermal effects arising mainly from thermal conduction through the bubble and the surrounding liquid can have significant effects on the total pressure, as has been demonstrated in various studies (e.g. see Chapman and Plesset [1971] ; Nigmatulin et al. [1981] ; Miksis and Ting [1984] ; Prosperetti [1991] ; Brennen [1995] ; Hao and Prosperetti [1999] and references therein). In particular, recent investigations by Nigmatulin et al. [1981] ; Prosperetti [1991] and Hao and Prosperetti [1999] have shown that the use of the polytropic law for the expansion (compression) of the gas, which neglects energy dissipation due to thermal conduction (thermal

damping), leads to considerable disagreement between theory and experiment in nonlinear oscillations of bubbles. It has also been recently demonstrated by Wang and Brennen [1998] and by Delale et al. [2001] that the use of the polytropic law for the expansion of the gas in steady-state cavitating nozzle flows can result in instabilities in the flow. Here we concentrate on the effect of thermal damping on bubble dynamics, especially on the instabilities mentioned. For this reason we consider the diffusion equation for a spherical vapor-gas bubble with low vapor concentration in the uniform pressure approximation in a similar manner as employed by Prosperetti [1991] for a gas bubble. The bubble wall temperature is fixed by the Plessset-Zwick [1952] solution of the heat equation in the surrounding liquid in the thin boundary layer approximation. Consequently, a general expression for the variation of the bubble pressure, that depends on the variation of the bubble wall temperature and its coupling to bubble dynamics, is obtained. The resulting equation is then coupled to the quasi-one-dimensional nozzle flow equations to determine the effect of thermal damping. Results obtained for quasi-one-dimensional steady-state cavitating nozzle flows show that the flashing flow instabilities discussed by Wang and Brennen [1998] and by Delale et al. [2001] are stabilized with or without the occurrence of bubbly shock waves in this thermal damping model. 


\section{Model equations for thermal damping in quasi-one-dimensional cavitating nozzle flows}

In this section we consider the model equations for unsteady quasi-one-dimensional nozzle flow equations. In particular, we consider the normalized equations of the latter where the classical Rayleigh-Plesset equation for bubble dynamics is modified to take into account bubble/bubble interactions (e.g. see Delale et al. [2001] ). These equations read

$$
\begin{gathered}
\rho=1-\beta, \\
A \frac{\partial \rho}{\partial t}+\frac{\partial}{\partial x}(\rho u A)=0, \\
\rho \frac{d u}{d t}=-\frac{\partial p}{\partial x}, \\
R^{3\left(\frac{1-\beta}{\beta}\right)}=\frac{1-\beta_{0}}{\beta_{0}}=k_{0}^{3}
\end{gathered}
$$

and

$$
\begin{gathered}
\frac{p_{b}-p}{L^{2}}=\frac{\left[1+\left(3 \Lambda^{2}-1\right)\left(R / k_{0}\right)^{3} / 2\right]}{\left[1+\left(R / k_{0}\right)^{3}\right]} R \frac{d^{2} R}{d t^{2}} \\
+\frac{3}{2} \frac{\left[1+2\left(2 \Lambda^{2}-1\right)\left(R / k_{0}\right)^{3}+\Lambda^{2}\left(R / k_{0}\right)^{6}\right]}{\left[1+\left(R / k_{0}\right)^{3}\right]^{2}}\left(\frac{d R}{d t}\right)^{2} \\
+\frac{S_{0}}{L^{2} R}+\frac{4}{L^{2}(R e) R} \frac{d R}{d t}
\end{gathered}
$$

with

$$
S_{0}=\frac{2 S^{\prime}}{p_{0}^{\prime} R_{0}^{\prime}} \quad, \quad R e=\frac{H_{0}^{\prime} \sqrt{p_{0}^{\prime} / \rho_{\ell}^{\prime}}}{\nu_{\ell}^{\prime}}
$$

where $\beta$ denotes the void fraction, $S^{\prime}$ is the surface tension at the liquid temperature $T_{0}^{\prime}, \rho_{\ell}^{\prime}$ is the liquid density at the liquid temperature $T_{0}^{\prime}, \nu_{\ell}^{\prime}$ is the corresponding liquid kinematic viscosity, $H_{0}^{\prime}$ is the nozzle inlet height, $R_{0}^{\prime}$ is the equilibrium radius at the nozzle inlet and $L=R_{0}^{\prime} / H_{0}^{\prime}$ is the ratio of micro to macro length. The normalized mixture pressure $p$, total bubble pressure $p_{b}$, mixture density $\rho$, mixture flow speed $u$ and bubble radius $R$ entering the system of equations (1)-(6) are defined by

$$
p=\frac{p^{\prime}}{p_{0}^{\prime}}, p_{b}=\frac{p_{b}^{\prime}}{p_{0}^{\prime}}, \rho=\frac{\rho^{\prime}}{\rho_{\ell}^{\prime}}=1-\beta, u=\frac{u^{\prime}}{\sqrt{p_{0}^{\prime} / \rho_{\ell}^{\prime}}} \quad \text { and } \quad R=\frac{R^{\prime}}{R_{0}^{\prime}}
$$

with $p_{0}^{\prime}$ denoting the inlet mixture pressure. The nozzle area, the nozzle axial coordinate and the time are normalized as

$$
A=\frac{A^{\prime}}{A_{0}^{\prime}} \quad, \quad x=\frac{x^{\prime}}{H_{0}^{\prime}} \quad \text { and } \quad t=\frac{t^{\prime}}{\Theta^{\prime}}=\frac{t^{\prime} \sqrt{p_{0}^{\prime} / \rho_{\ell}^{\prime}}}{H_{0}^{\prime}} .
$$

with $A_{0}^{\prime}$ denoting the nozzle inlet area. The parameter $\Lambda$, which enters the modified Rayleigh-Plesset equation (5), is the bubble/bubble interaction parameter that shows the range of influence of any bubble interacting with the rest and the normalized total derivative is $d / d t=\partial / \partial t+u \partial / \partial x$ (for details, see Delale et al. 2001). The system of eqs. (1)-(5) can now be supplemented by the normalized energy equation for the bubble interior together with the equation for the total bubble pressure in the uniform bubble pressure approximation and in the limit of low vapor concentration within the bubble following the derivation given by Prosperetti [1991] for a complete description of the model cavitating nozzle flow equations. These supplementary normalized equations are

$$
\frac{p_{b}}{T}\left\{\frac{d T}{d t}+\frac{D}{p_{b} R^{2}}\left[\lambda_{b}(T) \frac{\partial T}{\partial y}-\left.y \frac{\partial T}{\partial y}\right|_{y=1}\right] \frac{\partial T}{\partial y}\right\}
$$




$$
=\frac{\left(\gamma_{b}-1\right)}{\gamma_{b}} \frac{d p_{b}}{d t}+\frac{D}{R^{2} y^{2}} \frac{\partial}{\partial y}\left[y^{2} \lambda_{b}(T) \frac{\partial T}{\partial y}\right]
$$

and

$$
\frac{d}{d t}\left[R^{3 \gamma_{b}} p_{b}\right]=\left.3 \gamma_{b} R^{\left(3 \gamma_{b}-2\right)} D \frac{\partial T}{\partial y}\right|_{y=1}
$$

where the normalized bubble radial coordinate $y$ and the coefficient $D$ are defined by

$$
y=\frac{r^{\prime}}{R^{\prime}\left(t^{\prime}\right)}
$$

and

$$
D=\frac{\left(\gamma_{b}-1\right) \lambda_{b R}^{\prime} T_{0}^{\prime} \Theta^{\prime}}{\gamma_{b} p_{0}^{\prime} R_{0}^{\prime 2}}
$$

and where $T=T^{\prime} / T_{0}^{\prime}$ is the normalized bubble temperature distribution, $\gamma_{b}$ is the adiabatic exponent of the bubble, $\lambda_{b}(T)$ is the normalized thermal conductivity of the bubble content (normalized with respect to its value $\lambda_{b R}^{\prime}$ at the bubble wall and at the cold liquid temperature $T_{0}^{\prime}$ ). Equations (1)-(10) now constitute a complete system for $p, \rho, \beta, u, T, p_{b}$ and $R$ for a given normalized area $A(x)$ with appropriate initial and boundary conditions. The solution of such a problem is a difficult one, even if eqs. (9) and (10) are replaced by a polytropic law for the expansion of the gas, the latter law has been shown to be insufficient (e.g. see Nigmatulin et al. 1981, Prosperetti 1991). Therefore, approximations leading to certain simplifications, especially of eqs. (9) and (10), are needed so that the solution can be tractable, at least, by numerical means. A major difficulty in the coupling of eqs. (9) and (10) to the rest of the flow equations is the $y$ dependence of the term $1 / T d T / d t$ in eq. (9). However, a close examination shows that the term $1 / T d T / d t$ is only a slowly varying function of $y$ for $0<y<1$ and can, therefore, be taken to be independent of $y$. This assumption is equivalent to approximate the temperature field $T$ as a product of a function of $y$ by a function of $x$ and $t$. With this assumption, the integration of the $y$ dependence of the temperature in eq. (9) can be possible. In the limit of low vapor concentration eqs. (9) and (10) then reduce to

$$
\frac{\partial^{2} T}{\partial y^{2}}-\frac{1}{2 T}\left(\frac{\partial T}{\partial y}\right)^{2}+\left[\frac{2}{y}+\frac{y c}{T^{3 / 2}}\right] \frac{\partial T}{\partial y}-\frac{3}{T^{1 / 2}} c=0
$$

where we have defined $c(x, t)$ by

$$
c(x, t)=\left.\frac{\partial T}{\partial y}\right|_{y=1}
$$

and we have used the kinetic theory result $\lambda_{b}=\sqrt{T}$ for the normalized thermal conductivity $\lambda_{b}$. The partial differential equation (13) does not contain the time derivative and depends on the time $t$ and the space $x$ implicitly only through the function $c(x, t)$. It can, therefore, be treated as an ordinary differential equation in $y$ at any fixed $x$ and $t$. Furthermore, the temperature field at any field point $(x, t)$ should satisfy the boundary conditions

$$
\left.\frac{\partial T}{\partial y}\right|_{y=0}=0
$$

and

$$
\begin{gathered}
T(y=1, x, t)=T_{R}(x, t) \\
=1-\mathcal{L} \int_{0}^{t} \frac{R^{2}(x, \xi)(d R / d \xi) d \xi}{\left[\int_{\xi}^{t} R^{4}(x, \tau) d \tau\right]^{1 / 2}}
\end{gathered}
$$

where we have used the normalized form of the Plesset-Zwick formula [1952] for $T_{R}$ (from the solution of the energy equation in the surrounding liquid in the thin-boundary value approximation), with the integration in eq. (16) to be understood as being carried out following bubble motion and where $\mathcal{L}$ is defined by

$$
\mathcal{L}=\left(\frac{L^{\prime}}{T_{0}^{\prime} c_{p \ell}^{\prime}}\right)\left(\frac{\rho_{v}^{\prime}}{\rho_{\ell}^{\prime}}\right) \frac{R_{0}^{\prime}}{\left(\pi \Theta^{\prime} \alpha_{\ell}^{\prime}\right)^{1 / 2}}
$$


with $L^{\prime}$ denoting the latent heat of vaporization, $\rho_{v}^{\prime}$ denoting the saturated vapor density at the bubble wall temperature $T_{R}^{\prime}, c_{p \ell}^{\prime}$ and $\alpha_{\ell}^{\prime}$ denoting, respectively, the specific heat of the liquid at constant pressure and the thermal diffusivity of the liquid. An analytical solution to the boundary value problem given by eqs. (13), (15) and (16) then leads to the result

$$
c(x, t)=-\kappa \mathcal{L} \int_{0}^{t} \frac{R^{2}(x, \xi)(d R / d \xi) d \xi}{\left[\int_{\xi}^{t} R^{4}(x, \tau) d \tau\right]^{1 / 2}}
$$

where $\kappa \geq 0$ is a parameter to be determined from experiment. The polytropic law employed previously for the partial gas pressure can now be replaced by the law

$$
\frac{d}{d t}\left[R^{3 \gamma_{b}} p_{b}\right]=3 \gamma_{b} R^{\left(3 \gamma_{b}-2\right)} D c
$$

for the bubble pressure $p_{b}$ where $c$ is given by eq. (18). Equations (18) and (19) together with eqs. (1)-(6) for the unsteady nozzle flow equations now form the model equations for quasi-one-dimensional cavitating nozzle flows with thermal damping.

\section{Some applications and results}

In this section we investigate the effects of thermal damping on some cavitating nozzle flow solutions by the model developed above. In particular, we consider the effect of thermal damping on the flashing flow instabilities encountered in the quasi-one-dimensional steady-state nozzle flow solutions obtained by direct numerical simulation by Wang and Brennen [1998] and by solving a third order dynamical system of scaled variables for the flow speed by Delale et al. [2001]. As for the nozzle, we use the slender nozzle employed by Delale et al. [2001] with origin $(x=0)$ at the throat and inlet at $x=-\ell$. For steady-state, eq. (19) integrates to

$$
p_{b}(x)=\left[1+S_{0}+3 \gamma_{b} D \int_{-\ell}^{x} \frac{[R(\xi)]^{\left(3 \gamma_{b}-2\right)}}{u(\xi)} c(\xi) d \xi\right] /[R(x)]^{3 \gamma_{b}}
$$

where $c$, given by eq. (18), takes the form

$$
c(x)=-\kappa \mathcal{L} \int_{-\ell}^{x} \frac{R^{2}(\xi)(d R / d \xi) d \xi}{\left\{\int_{\xi}^{x}\left[R^{4}(z) / u(z)\right] d z\right\}^{1 / 2}}
$$

with $\kappa \geq 0$. We can now implement eqs. (20) and (21) in the steady-state solution of Delale et al. (2001) replacing the polytropic law for the gas pressure.

We consider a bubbly cavitating flow of water-vapor/air bubbles in water at $20^{\circ} \mathrm{C}$, with a constant partial vapor pressure $p_{v}^{\prime}=0.074$ bar, a constant surface tension coefficient $S^{\prime}=7.1 \times 10^{-2} \mathrm{~N} / \mathrm{m}$ and a constant water viscosity $\mu_{\ell}^{\prime}=10^{-3} \mathrm{~kg} / \mathrm{m}$-s, through the converging-diverging nozzle shown in figure 1 . The initial cavitation number $\sigma_{0}$, the initial void fraction $\beta_{0}$ and the initial flow speed $u_{0}^{\prime}$ are fixed at the values $\sigma_{0}=0.5, \beta_{0}=10^{-5}$ and $u_{0}^{\prime}=10 \mathrm{~m} / \mathrm{s}$ respectively. The initial radius $R_{0}^{\prime}$ is varied between $10 \mu \mathrm{m}$ and $33 \mu \mathrm{m}$. When the partial gas pressure is approximated by the polytropic law leaving out the thermal damping effects on the flow field, it has already been demonstrated by Delale et al. [2001] that, under the conditions stated above, stable steady-state cavitating nozzle flow solutions in the quasi-one-dimensional approximation are possible if the initial radius $R_{0}^{\prime}$ is below some critical value (for the conditions stated above, this critical value is $10.22 \mu \mathrm{m}$ ). When the initial radius exceeds this critical value with the rest of the conditions held fixed, flashing flow instabilities, similar to those obtained by Wang and Brennen [1998], are observed. In this work we replace the polytropic law for the gas pressure by eqs. (20) and (21) to include the damping effect of heat conduction through the bubble, which has already been demonstrated to be quite significant for oscillating gas bubbles by Nigmatulin et al. [1981] and by Prosperetti [1991]. Since bubble/bubble interactions become important only when the bubbles grow to appreciable size, it is 


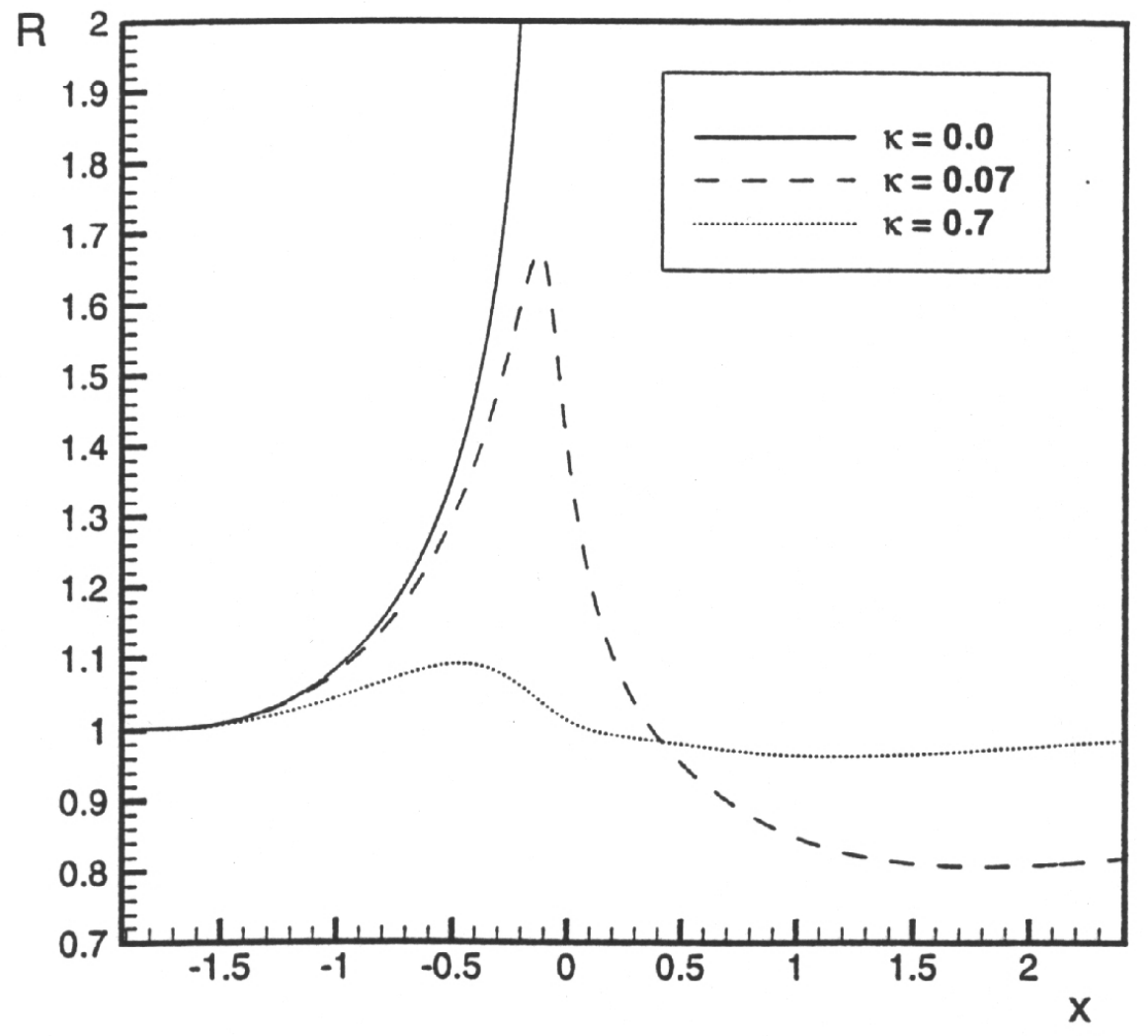

Figure 1: Distributions of the normalized bubble radius $R$ without $(\kappa=0.0)$ and with thermal damping $(\kappa=0.07$ and $\kappa=0.7)$ along the nozzle axis (with throat at $x=0$ with initial void fraction $\beta_{0}=10^{-5}$ , initial cavitation number $\sigma_{0}=0.5$, inlet flow speed $u_{0}^{\prime}=10 \mathrm{~m} / \mathrm{s}$ and initial radius $R_{0}^{\prime}=10 \mu \mathrm{m}$ for the steady-state solution with $\Lambda=1$ of water vapor-air bubbles in water at $20^{\circ} \mathrm{C}$. 


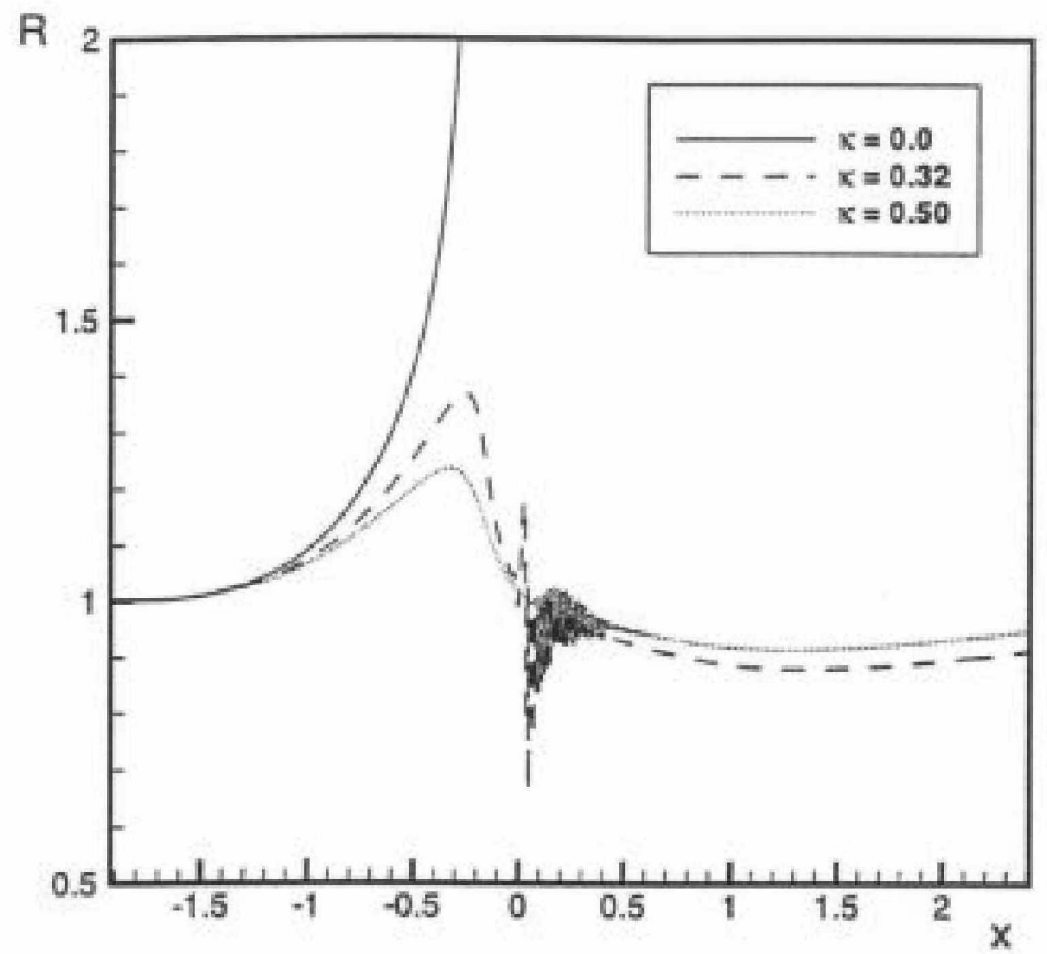

Figure 2: Distributions of the normalized bubble radius $R$ without $(\kappa=0.0)$ and with thermal damping ( $\kappa=0.32$ and $\kappa=0.5$ ), corresponding to bubbly shock solutions, along the nozzle axis (with throat at $x=0$ ) with initial void fraction $\beta_{0}=10^{-5}$, initial cavitation number $\sigma_{0}=0.5$, inlet flow speed $u_{0}^{\prime}=10$ $\mathrm{m} / \mathrm{s}$ and initial radius $R_{0}^{\prime}=33 \mu \mathrm{m}$ for the steady-state solution with $\Lambda=1$ of water vapor-air bubbles in water at $20^{0} \mathrm{C}$. 
natural to think that thermal damping effects would be more significant prior to these interaction effects so that we can set $\Lambda=1$ (this corresponds to employing the Rayleigh-Plesset equation). Figure 1 shows the thermal damping effect on the normalized radius $R$ for the case with initial radius $R_{0}^{\prime}=20 \mu \mathrm{m}$ where flow instabilities were observed using the polytropic law for the gas pressure ( the case $\kappa=0$ ). Thermal damping seems to reduce the normalized maximum radius, the reduction being greater when the coefficient $\kappa$ assumes higher values (e.g. for $\kappa=0.7$ ). When the initial radius $R_{0}^{\prime}$ is increased even further (e.g. to a value of $R_{0}^{\prime}=33 \mu \mathrm{m}$ ) with the rest of the nozzle inlet conditions kept fixed, the flashing flow instabilities become even stronger with much steeper gradients along the nozzle axis. In this case larger values of the thermal damping parameter $\kappa$ are required and such instabilities are usually stabilized by bubbly shock solutions. Figure 2 demonstrates the thermal damping effect by a bubbly shock solution on the normalized radius distribution $R$ as the parameter $\kappa$ is increased from zero to 0.5. The increase in the presure coefficient with $\kappa$ can be identified as an increase in the strength of the bubbly shock. In conclusion, the instabilities observed in quasi-steady nozzle flows are seen to be stabilized by the present thermal damping model with or without the occurrence of bubbly shock waves.

\section{Acknowledgements}

This work is supported in part by the National Science Foundation under contract number CTS9979258 at Caltech, in part by the Scientific and Technical Research Council of Turkey (TÜBİTAK) and in part by the Turkish Academy of Sciences (TÜBA). The author is indebted to Professors C.E.Brennen and T.Colonius for many illuminating comments and discussions.

\section{References}

BRENNEN, C.E. (1995) Cavitation and Bubble Dynamics, Oxford University Press

CHAPMAN, R.P. \& PLESSET, M.S. (1971) Thermal effects in the free oscillation of gas bubbles. ASME J. Basic Eng. 93, 373-376.

DELALE, C.F. , SCHNERR, G.H. \& SAUER, J. (2001) Quasi-one-dimensional steady-state cavitating nozzle flows. J. Fluid Mech. 427, 167-204.

HAO, Y. \& PROSPERETTI, A. (1999) The dynamics of vapor bubbles in acoustic pressure fields. Phys. Fluids 11, 2008-2019.

MIKSIS, M.J. \& TING, L. (1984) Nonlinear radial oscillations of a gas bubble including thermal effects. J. Acoust. Soc. Am. 76, 897-905.

NIGMATULIN, R.I., KHABEEV, N.S. \& NAGIEV, F.B. (1988) Dynamics, heat and mass transfer of vapourgas bubbles in a liquid. Int. J. Heat Mass Transfer 24, 1033-1044.

PLESSET, M.S. \& ZWICK, S.A. (1952) A nonsteady heat diffusion problem with spherical symmetry. J. Appl. Physics 23, 95-98.

PROSPERETTI, A. (1991) The thermal behavior of oscillating gas bubbles. J. Fluid Mech. 222, $587-616$. WANG, Y.C. \& BRENNEN, C.E. (1998) One-dimensional bubbly cavitating flows through a convergingdiverging nozzle. ASME J. Fluids Eng. 120, 166-170. 